LA UR-96-93

\title{
CRITICAL EXPONENTS OF THE 3-D ISING MODEL
}

\author{
Rajan Gupta* $§$ \\ T-8, MS-B285, Los Alamos National Laboratory, Los Alamos, NM 87545 \\ Pablo Tamayo ${ }^{\dagger}$ \\ T-8, MS-B285, Los Alamos National Laboratory, Los Alamos, NM 87545 \\ and \\ Thinking Machines Corporation, Cambridge, MA 02143, USA
}

\begin{abstract}
We present a status report on the ongoing analysis of the 3D Ising model with nearest-neighbor interactions using the Monte Carlo Renormalization Group (MCRG) and finite size scaling (FSS) methods on $64^{3}$, $128^{3}$, and $256^{3}$ simple cubic lattices. Our MCRG estimates are $K_{n n}^{c}=0.221655(1)(1)$ and $\nu=0.625(1)$. The FSS results for $K^{c}$ are consistent with those from MCRG but the value of $\nu$ is not. Our best estimate $\eta=0.025(6)$ covers the spread in the MCRG and FSS values. A surprise of our calculation is the estimate $\omega \approx 0.7$ for the correction-to-scaling exponent. We also present results for the renormalized coupling $g_{R}$ along the MCRG flow and argue that the data support the validity of hyperscaling for the 3D Ising model.
\end{abstract}

Date $=1 / 14 / 96$

* Invited talk presented at the US-Japan Bilateral Seminar, Maui, August 28-31, 1995 . To be published in International Journal of Modern Physics C.

$\S$ rajan@qcd.lanl.gov

† tamayo@think.com. 


\section{Introduction}

The 3D Ising model has, over the last 25 years, been used to test the accuracy of various analytical and numerical methods for solving Statistical Mechanics systems. In 1992 we presented results of simulations on $64^{3}$ and $128^{3}$ lattices using the Monte Carlo Renormalization Group (MCRG) method 值. While that work improved on previous MCRG estimates [2] [3]), it left us with four unanswered questions. The first and most tantalizing was -- are the exponents rational numbers, i.e. $\nu=0.625$ and $\eta=0.025$. Second, a more precise determination of the corrections-to-scaling exponent is needed as it is the largest source of systematic errors. Third, we wanted to resolve/understand the differences between our MCRG and existing finite size scaling/ $\epsilon$-expansion results. Lastly, we wanted to investigate whether hyperscaling holds for this model. This talk is a summary of the current status of our calculations.

In order to address these issues we have extended the calculations in the following ways. We have made higher statistics runs on $64^{3}, 128^{3}$, and $256^{3}$ lattices at $K=0.221652$ and 0.221655 . On these lattices we have evaluated, in addition to correlation functions needed for MCRG studies, quantities needed for finite size scaling (FSS) analysis and the calculation of the renormalized coupling $g_{R}$. As a result we have better estimates of the critical coupling $K_{n n}^{c}$, the exponents $\nu$ and $\eta$ from both MCRG and finite-size scaling (including histogram re-weighting) analysis, and can address the issue of hyperscaling violations. Our new estimate of the corrections-to-scaling exponent $\omega \approx 0.7$ is significantly smaller than that from other methods.

All the simulations have been done on the Thinking Machines CM-5 (at the ACL at LANL) and CM-5E (at TMC) computers. We used the Swendsen-Wang cluster update algorithm 四 and a 250-long 64-bit wide shift-register (Kirkpatrick-Stoll) random number generator in each vector unit. The new results agree with our previous calculation and those in [2] and [3]. Each of these calculations used a different random number generator, so their consistency suggests that there is no obvious bias in the sequence of random numbers generated (see P. Coddington's talk on random number generators at this workshop). Our most extensive results are at $K_{n n}=0.221655$, which is our present best estimate of $K_{c}$, and the statistical sample consists of $600 \mathrm{~K}, 500 \mathrm{~K}$, and $400 \mathrm{~K}$ measurements on $64^{3}, 128^{3}$, and $256^{3}$ lattices respectively.

The details of our implementation of the MCRG method are the same as in [1]. The only change is that we have added 3 more even couplings (for a total of 56) and one more odd couplings (total 47). The original 53 even and 46 odd couplings were contained in either a $3 \times 3$ square or a $2^{3}$ template [1]. The new couplings are those obtained by adding a fourth spin along the cartesian axis to the $3 \times 3$ template.

We store the magnetization and energy for each configuration, from which we can calculate quantities like the specific heat, susceptibility, Binder's cumulant $U=3-\left\langle m^{4}\right\rangle /\left\langle m^{2}\right\rangle^{2}$, etc.. These results are then evaluated as a function of $K$, in a small neighborhood of $K_{\text {simulation }}$, using the histogram re-weighting method [5]. The finite size analysis of these quantities follows the work of Ferrenberg and Landau [6], i.e. without corrections to scaling terms. To calculate $g_{R}$, we also need the finite lattice correlation length $\xi$. This is calculated in two ways:

$$
\begin{aligned}
\left\langle\sum_{x, y} s(x, y, z) \sum_{x, y} s(x, y, 0)\right\rangle & \underset{z \rightarrow \infty}{\longrightarrow} a e^{-z / \xi}, \\
\frac{1}{k^{2}}\left(\frac{S(0)^{2}}{S(k)^{2}}-1\right) & =\xi^{2},
\end{aligned}
$$

where $S(k)=\sum_{x, y, z} s(x, y, z) e^{i \vec{k} \cdot \vec{x}}$. We investigate the 5 lowest momenta but present results only for the lowest, $k_{z}=2 \pi / L$, as it has the best signal. With $\xi$ in hand we calculate $g_{R}$ defined as [7]

$$
g_{R}(K, L)=\left(\frac{L}{\xi}\right)^{d}\left(3-\frac{\left\langle m^{4}\right\rangle}{\left\langle m^{2}\right\rangle^{2}}\right) .
$$

This is expected to scale as

$$
g_{R}(K, L) \sim L^{-w^{*}}
$$

where $w^{*}=(\gamma+d \nu-2 \Delta) / \nu$ is referred to as the anomalous dimension of the vacuum. If hyperscaling holds then $g_{R}^{*} \rightarrow$ finite non-zero constant as $L \rightarrow \infty$ and $K \rightarrow K^{*}$.

For the purpose of error analysis the data have been divided into bins of size 10,000 measurements. All errors are then calculated by a single elimination jackknife procedure over these bins. This talk is organized as follows. We first summarize the MCRG results, then compare them with FSS estimates, and finally give data for $g_{R}$, both at $K_{\text {sim }}=0.221655$ and along the MCRG flow, and discuss hyperscaling. 


\section{Nearest-Neighbor Critical Coupling $K_{n n}^{c}$}

We calculate $K_{n n}^{c}$ using the two lattice $M C R G$ method. The results for the two starting couplings $K_{n n}=0.221652$ and 0.221655 and for the two pairs of lattices, $256^{3}$ versus $128^{3}$ and $128^{3}$ versus $64^{3}$, are shown in Table 1, where for ease of comparison we have also reproduced results from [1]. We expect the deviations $K^{c}(\infty)-K^{c}(n)$, where $n$ is the blocking step, to converge by the geometric factor $\lambda_{u} / \lambda_{t}$ as a function of the blocking level $n$. For the 3D Ising model $\lambda_{u} / \lambda_{t} \approx 6$, and our data for $K_{n n}^{c}$ (see Table 1) do roughly show convergence by this factor.

For both starting couplings the data show that the new estimates from the $128^{3} / 64^{3}$ lattices analysis are consistent with our earlier results and give $K_{n n}^{c}=0.221652$. However, the $256^{3} / 128^{3}$ results show better convergence with respect to blocking steps, and also show a systematic shift at all blocking levels compared to the $128^{3} / 64^{3}$ data. Our new estimate from $256^{3} / 128^{3}$ comparison is $K_{n n}^{c}=0.221655 \pm 0.000001$ where only the statistical error has been quoted. This shift in $K_{n n}^{c}$ with lattice size shows that there could still be finite size corrections at the level of statistical errors, i.e. a systematic error of +0.000001 . So our final best estimate is

$$
K_{n n}^{c}=0.221655 \pm 0.000001_{-0}^{+0.000001},
$$

which is consistent with the recent result 0.2216546(10) obtained using FSS analysis by Blöte et al. [8]. In view of this we take the results at $K_{n n}=0.221655$ to represent the critical point values.

\begin{tabular}{|c|c|c|c|c|c|c|}
\hline Level & 0.2216550 & 0.2216520 & 0.2216550 & 0.2216540 & 0.2216520 & 0.2216440 \\
$n / m$ & $256^{3} / 128^{3}$ & $256^{3} / 128^{3}$ & $128^{3} / 64^{3}$ & $128^{3} / 64^{3}$ & $128^{3} / 64^{3}$ & $128^{3} / 64^{3}$ \\
& $N e w$ & $N e w$ & New & 四 & New & 四 \\
\hline $2 / 1$ & $217 \pm 11$ & $184 \pm 15$ & $095 \pm 13$ & $070 \pm 16$ & $046 \pm 23$ & $095 \pm 17$ \\
\hline $3 / 2$ & $469 \pm 9$ & $458 \pm 18$ & $413 \pm 15$ & $406 \pm 18$ & $394 \pm 27$ & $417 \pm 22$ \\
\hline $4 / 3$ & $537 \pm 10$ & $534 \pm 21$ & $504 \pm 16$ & $500 \pm 21$ & $501 \pm 31$ & $508 \pm 24$ \\
\hline $5 / 4$ & $549 \pm 10$ & $549 \pm 22$ & $523 \pm 16$ & $514 \pm 26$ & $516 \pm 37$ & \\
\hline $6 / 5$ & $547 \pm 10$ & & & & & \\
\hline
\end{tabular}

Table 1. Estimates of $K_{n n}^{c}$ as a function of the blocking level and $K_{\text {simulation. For brevity only the last }}$ three decimal places have been quoted, so $547 \pm 10$ is short for $0.2216547 \pm 0.0000010$. We have included our old data from [1] to facilitate comparison. The highest blocked lattice is $4^{3}$ except at $K_{n n}=0.221652$ where it is only $8^{3}$ on $128^{3}$ and $256^{3}$ lattices. The quoted errors are the statistical errors after averaging the data over the 56 even operators. The data show a systematic shift between the $256^{3} / 128^{3}$ and $128^{3} / 64^{3}$ lattices.

\section{Correlation length exponent $\nu$}

The correlation length exponent $\nu$ is determined from the leading even eigenvalue $\lambda_{t}$ of the linearized transformation matrix $\mathcal{T}_{\alpha \beta}^{n}$,

$$
y_{t} \equiv \frac{1}{\nu}=\frac{\ln \lambda_{t}}{\ln b},
$$

where $b=2$ is the scale factor of the majority rule blocking transformation. Our preferred data for $\lambda_{t}$ $\left(K_{\text {sim }}=0.221655\right)$ is shown in Table 2 as a function of the blocking step and lattice size. There are three possible sources of systematic errors that affect the $L \rightarrow \infty$ and $K \rightarrow K_{c}$ estimates for $\lambda_{t}$. These are

1. The number of operators measured, i.e. the truncation errors in evaluating eigenvalues from a finite dimensional $\mathcal{T}_{\alpha \beta}^{n}$. We find that the number of operators needed to achieve convergence increases with the blocking level $n$. The data show that with the 56 even operators the eigenvalues show convergence at all levels. (The same is true in the sector of odd interactions from which we extract the exponent $\eta$ ). Even at the highest blocked level there is no detectable variation after including 30 operators. Unfortunately, 
the convergence with the number of operators is not monotonic and there is no independent way of confirming that the results have converged. Thus, we cannot estimate the possible error due to lack of convergence, and guess that it is smaller than the statistical error.

2. Finite size effects on blocked lattices. It has been observed in [1], [2], and [3] that finite size effects are discernible only when blocking from $8^{3} \rightarrow 4^{3}$ lattices or smaller. The correction increases the estimate of $\lambda_{t}$. In Ref. [3] the correction in $\lambda_{t}^{8 \rightarrow 4}$ was estimated to be 0.02 . Our estimate based on comparing $256^{3}, 128^{3}, 64^{3}$ lattices is $\approx 0.01$. Applying +0.01 as the correction to our $256^{3}$ lattices data, we get the lower limit $\lambda_{t}^{8 \rightarrow 4}=3.008$ corresponding to $\nu=0.6294$. We discuss the $L \rightarrow \infty$ limit below.

3. Error in the estimate of $K_{n n}^{c}$. The value of $\lambda_{t}$ also increases with $K_{n n}^{c}$ as shown by the data in Fig. 1, and in Ref. [2]. The dependence of $\lambda_{t}$ on $K_{n n}^{c}$ is marginal on the first couple of blocking levels and then increases rapidly with $n$ for $n>3$. Since our estimate of $K_{n n}^{c}$ is converging from below, our results at $K=0.221655$ may underestimate $\lambda_{t}$.

The bottom line is that the systematic effects discussed in items 2 and 3 will tend to increase $\lambda_{t}$ or equivalently decrease $\nu$.

\begin{tabular}{|c|c|c|c|c|c|c|}
\hline & \multicolumn{3}{|c|}{$\lambda_{t}$} & \multicolumn{3}{c|}{$\lambda_{h}$} \\
\hline & $256^{3}$ & $128^{3}$ & $64^{3}$ & $256^{3}$ & $128^{3}$ & $64^{3}$ \\
\hline $0 / 1$ & $2.681(2)$ & $2.685(2)$ & $2.684(3)$ & $5.4948(06)$ & $5.4941(06)$ & $5.4948(08)$ \\
$1 / 2$ & $2.843(2)$ & $2.847(2)$ & $2.847(2)$ & $5.5050(02)$ & $5.5052(02)$ & $5.5050(03)$ \\
$2 / 3$ & $2.930(3)$ & $2.930(3)$ & $2.930(3)$ & $5.5501(02)$ & $5.5499(03)$ & $5.5494(07)$ \\
$3 / 4$ & $2.969(3)$ & $2.973(4)$ & $2.971(5)$ & $5.5741(04)$ & $5.5745(08)$ & $5.5701(21)$ \\
$4 / 5$ & $2.995(5)$ & $2.985(7)$ & & $5.5845(11)$ & $5.5826(31)$ & \\
$5 / 6$ & $2.998(7)$ & & & $5.5850(31)$ & & \\
\hline
\end{tabular}

Table 2. Estimates of $\lambda_{t}$ and $\lambda_{h}$ as a function of the blocking level and lattice size for $K=0.221655$.
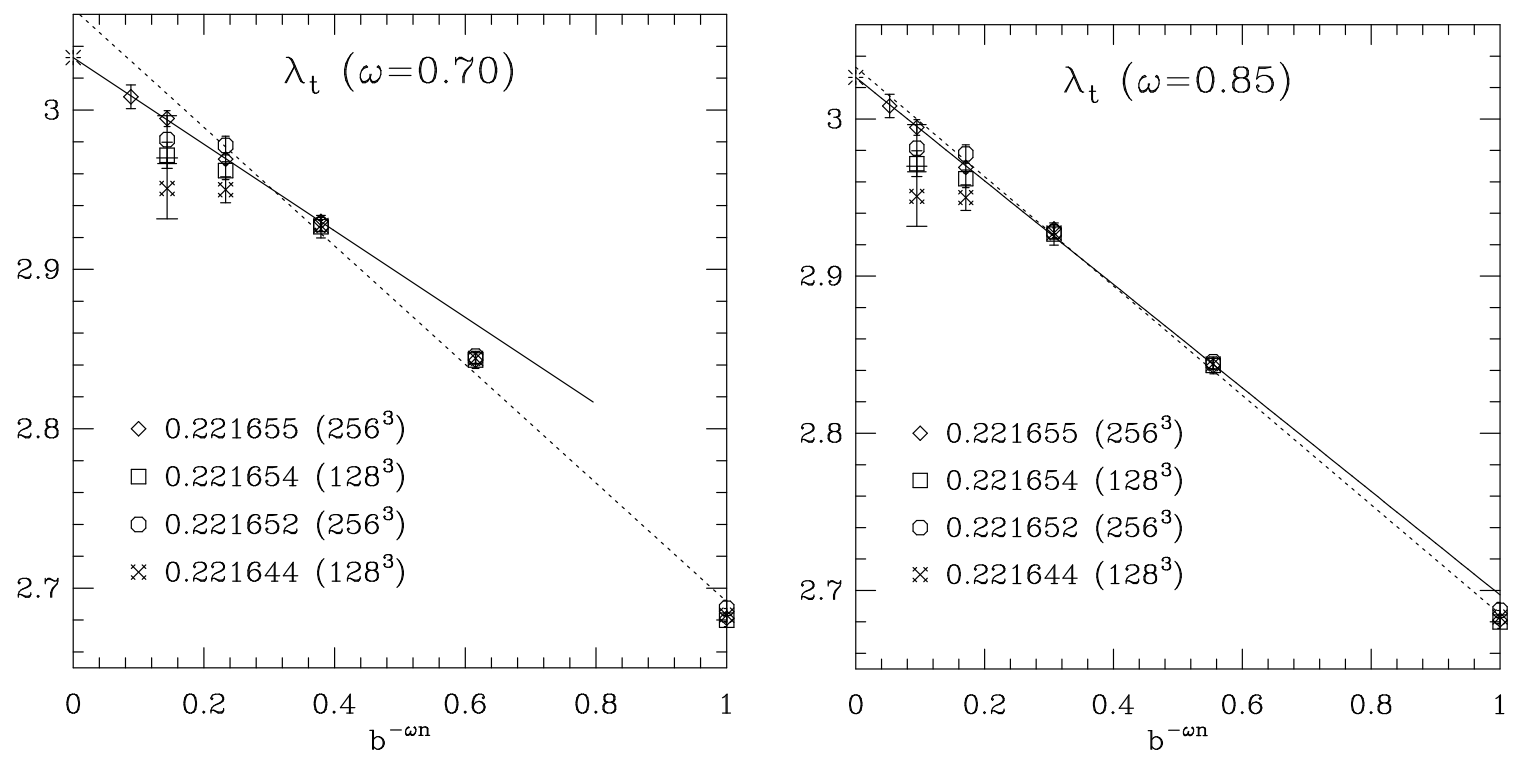

Fig. 1: Estimates of $\lambda_{t}$ as a function of the blocking level $n$ for simulations at different couplings $K$. The best estimate of the extrapolated value with $\omega=0.70(\omega=0.85)$ is from the linear fit to the diamonds skipping $n=0,1(n=0)$ points. The fit to all the diamond points is, for comparison, shown by the dotted line. 
Finally, we are interested in the value of $\lambda_{t}$ at the fixed point. To obtain this we extrapolate $\lambda_{t}$ versus the blocking level $n$ using [1]

$$
\lambda_{t}(n)=\lambda_{t}^{*}+a_{t} b^{-\omega n} .
$$

where $\omega=\theta / \nu$ is the leading correction-to-scaling exponent. There are two issues that need to be addressed in doing this extrapolation in the number of blocking steps $n$ (i.e. the $L \rightarrow \infty$ limit). The first is the value of $\omega$ and the second is whether the fit should exclude the first few blocking steps to avoid transients, to account for which requires further corrections to the leading behavior shown in Eq.(3.2). The calculation of $\omega$ is discussed in the next section and our present estimate $\omega=0.7$ is surprisingly low. We, therefore present an analysis for $\omega=0.7$ and 0.85 , where the second estimate is roughly what is given by other methods (FSS, $\epsilon$-expansion, etc. See [9] for a very recent survey). The question of transients is completely empirical, i.e. we neglect data at initial blocking steps until $\chi^{2} \sim 1$.

On basis of the quality of the fit to the $K=0.221655, L=256$ data the best estimates for the two extreme values of $\omega$ are

$$
\begin{array}{lll}
\lambda_{t}^{*}=3.028(3) & \Longrightarrow \quad \nu=0.6256(5) & (\omega=0.85, n=2-6) \\
\lambda_{t}^{*}=3.033(6) & \Longrightarrow \quad \nu=0.6247(10) & (\omega=0.70, n=3-6) .
\end{array}
$$

These two estimates are consistent, we therefore take the mean value and the larger of the two errors to get our present best estimate $\nu=0.625(1)$. To improve this result will require a better estimate of $\omega$ and data on larger lattices (more blocking steps).

\section{Correlation function exponent $\eta$}

The correlation function exponent $\eta$ is given by

$$
\eta=d+2-2 \frac{\log \lambda_{h}}{\log b} \equiv d+2-2 y_{h},
$$

where $b=2, d=3$ and $\lambda_{h}$ is the largest eigenvalue of $\mathcal{T}_{\alpha \beta}$ constructed from the odd interactions. The discussion of the type and sign of the various systematic errors in the extraction of $\lambda_{h}$ is the same as for $\lambda_{t}$. The raw data are shown in Table 2, and the value of finite size correction we apply to $\lambda_{h}^{8 \rightarrow 4}$ is 0.002 . Then, from the $L=256$ data $\left(\lambda_{h}^{8 \rightarrow 4}=5.587(3)\right)$ we get the upper bound $\eta=0.0359(16)$.

To extrapolate to $L \rightarrow \infty$ we proceed in exactly the same way as for $\lambda_{t}$. However, as exemplified by Fig. 2, the points at $n=4,5$ show significant deviations from the linear fits. Even though the fit with $\omega=0.85$ is somewhat better, the $n=5$ point raises questions about the validity of the linear extrapolation. There are two possibilities. One, the value flattens out at $\lambda_{h}=5.59$, in which case $\eta=0.034$. Second, the points at higher blocking levels are not well determined (note the large dependence of $\lambda_{h}$ on $K$ at higher $n$ as shown in Fig. 2. The systematic errors could therefore be larger than the statistical), and the linear extrapolation is valid. In the latter case one gets

$$
\begin{aligned}
& \lambda_{h}^{*}=5.603(4) \quad \Longrightarrow \quad \eta=0.028(2) \quad(\omega=0.85, n=2-5) \text {, } \\
& \lambda_{h}^{*}=5.610(5) \quad \Longrightarrow \quad \eta=0.024(3) \quad(\omega=0.70, n=2-5) \text {. }
\end{aligned}
$$

For errors we have used the difference between the extrapolated values with fits to $n=1-5$ and $n=2-5$ points. The bottom line is that even though we have improved the estimates for $\lambda_{h}$ on lattices of size up to $L=256$, there is still a large ambiguity in the determination of $\eta$ due to the extrapolation to $L=\infty$.

\section{Corrections-to-scaling exponent $\omega$}

It should be clear from the above discussion that a precise estimate of $\omega$ is very important in order to take the $L \rightarrow \infty$ limit. In a MCRG calculation the correction-to-scaling exponent is determined from the sub-leading eigenvalue $\lambda_{t, 2}$ in the even sector; $\omega \equiv-y_{t, 2}=-\log \lambda_{t, 2} / \log b$. Only if $\omega$ is known can the 

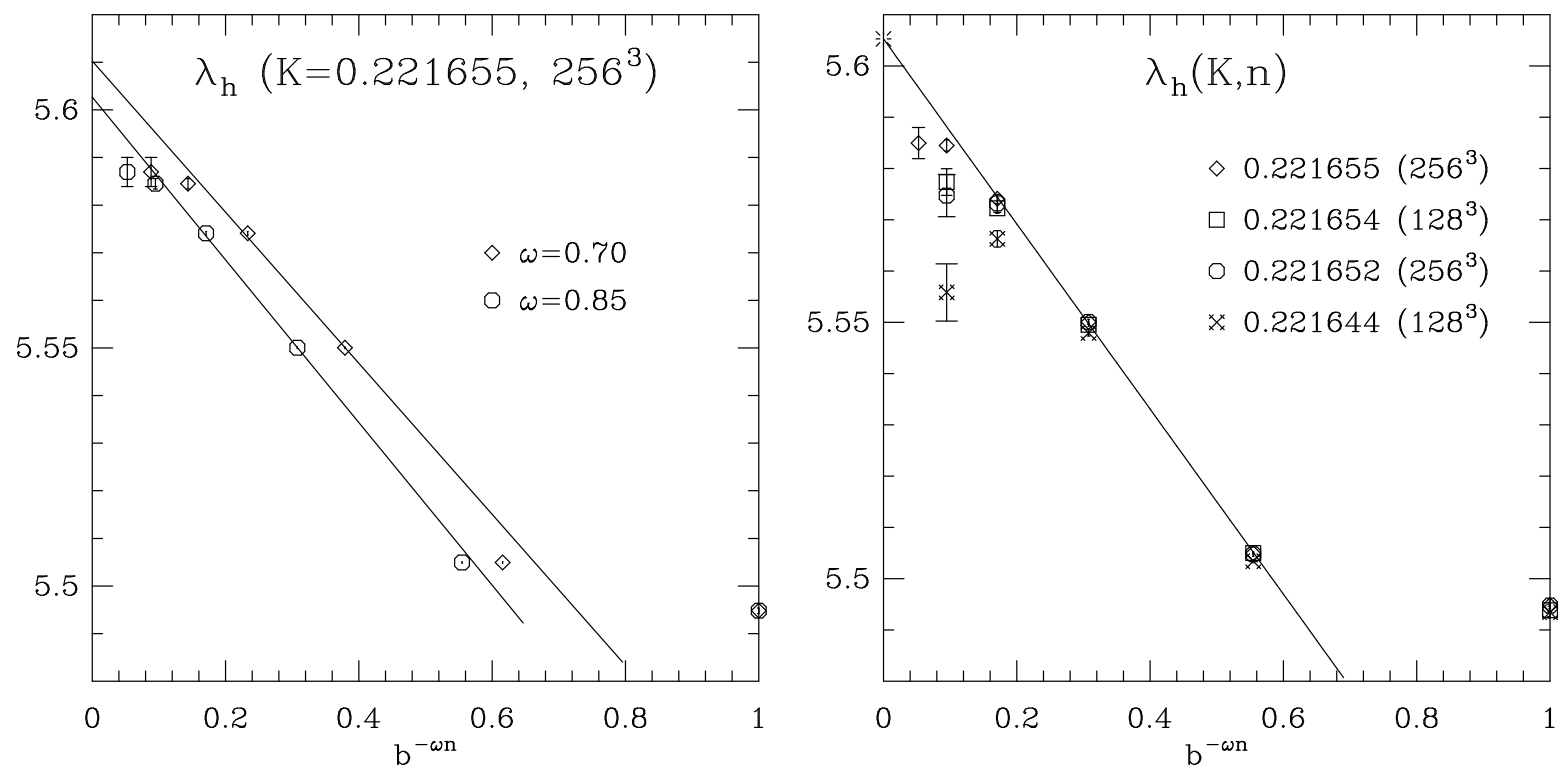

Fig. 2: (A) Estimates of $\lambda_{h}$ as a function of the blocking level $n$ for simulations on $256^{3}$ lattices at $K=0.221655$. The same data is plotted for $\omega=0.70$ and 0.85 , and for each case we show a linear fit to $n=2-5$ points. The $n=5$ point deviates significantly from the fits. (B) $\lambda_{h}$ as a function of $K$ and $n$.

exponents $\nu$ and $\eta$, calculated along a critical RG flow, be extrapolated to the fixed point using Eq. (3.2). We have therefore spent considerable effort in estimating $\omega$. With our best data, shown in Table 3, we have overcome the statistical problem that plagued the data in [1]. The second and third eigenvalue no longer merge into a complex pair when the number of operators is $\geq 15$. However, we now find that the value of $\omega$ decreases both with the number of operators and blocking steps; it starts off at $\approx 0.85$ on the first blocking step and with 10 operators, but finally settles down to

$$
\omega \sim 0.70 \Longrightarrow \theta=\omega \nu \sim 0.44 \text {. }
$$

This value is significantly smaller than the world average, $\theta=0.54(3)$, of estimates obtained using other methods as presented in [9]. Clearly, this issue requires further attention.

\begin{tabular}{|l|l|l|l|l|l|}
\hline & 10 Ops & 20 Ops & 30 Ops & 40 Ops & 50 Ops \\
\hline 1 & $0.90(3)$ & $1.01(4)$ & $0.97(4)$ & $0.98(4)$ & $0.97(4)$ \\
2 & $0.85(3)$ & $0.83(4)$ & $0.79(4)$ & $0.80(4)$ & $0.79(4)$ \\
3 & $0.81(3)$ & $0.76(4)$ & $0.73(4)$ & $0.72(4)$ & $0.73(4)$ \\
4 & $0.79(3)$ & $0.74(4)$ & $0.70(4)$ & $0.67(4)$ & $0.67(4)$ \\
5 & $0.75(3)$ & $0.73(3)$ & $0.70(3)$ & $0.70(3)$ & $0.70(3)$ \\
\hline
\end{tabular}

Table 3. Estimates of $\omega$ as a function of the blocking level and the number of operators used in constructing the transformation matrix $\mathcal{T}_{\alpha \beta}$. The data is for $K=0.221655$.

\section{Finite size scaling using the histogram re-weighting method.}

We use the histogram re-weighting method [5] to estimate (i) the position of the maximum for various thermodynamic quantities, (ii) the value at this maximum, and (iii) the value of $K$ at the point of crossing 
of $U$ and $g_{R}$ for two different size lattices. The method consists of building a histogram $H(E, m)$, i.e. the number of configurations with energy $E$ and magnetization $m$, using an equilibrium (canonical) Monte Carlo simulation at temperature $K_{\text {sim }}$. With this histogram, the equilibrium probability distribution at other temperatures $K$ is

$$
P_{K}(E, m)=\frac{H(E, m) \exp [\Delta K E]}{\sum_{E, m} H(E, m) \exp [\Delta K E]},
$$

where $\Delta K=K-K_{\text {sim }}$. The average value of any function of $E$ and $m, Q(E, m)$, at coupling $K$ is then given by

$$
\left\langle Q_{K}(E, m)\right\rangle=\sum_{E, m} Q(E, m) P_{K}(E, m) .
$$

The value of thermodynamic derivatives with respect to $K$ are obtained from Monte Carlo measurements of correlation functions,

$$
\frac{d\langle Q\rangle}{d K}=\langle Q E\rangle-\langle Q\rangle\langle E\rangle
$$

Again, by using the re-weighting technique these correlation functions can be evaluated at all temperatures in a certain neighborhood of $K_{\text {sim }}$. Thus, the location and magnitude of the peaks, and the points of crossings can be obtained from simulations at a single temperature.

The propagation of errors under this re-weighting is not straightforward and has been dealt with by Ferrenberg and by Swendsen in their talks at this meeting. In our current analysis the error estimates are the naive statistical ones and ignore all correlations and uncertainty in determining $H(E, m)$. We only present results for $K_{\text {sim }}=0.221655$ as these have higher statistics and correspond to our estimate of the infinite volume $K_{c}$. With this and the re-weighted data generated from it in hand we use the appropriate finite size scaling relations to derive estimates of the critical exponents and temperature.
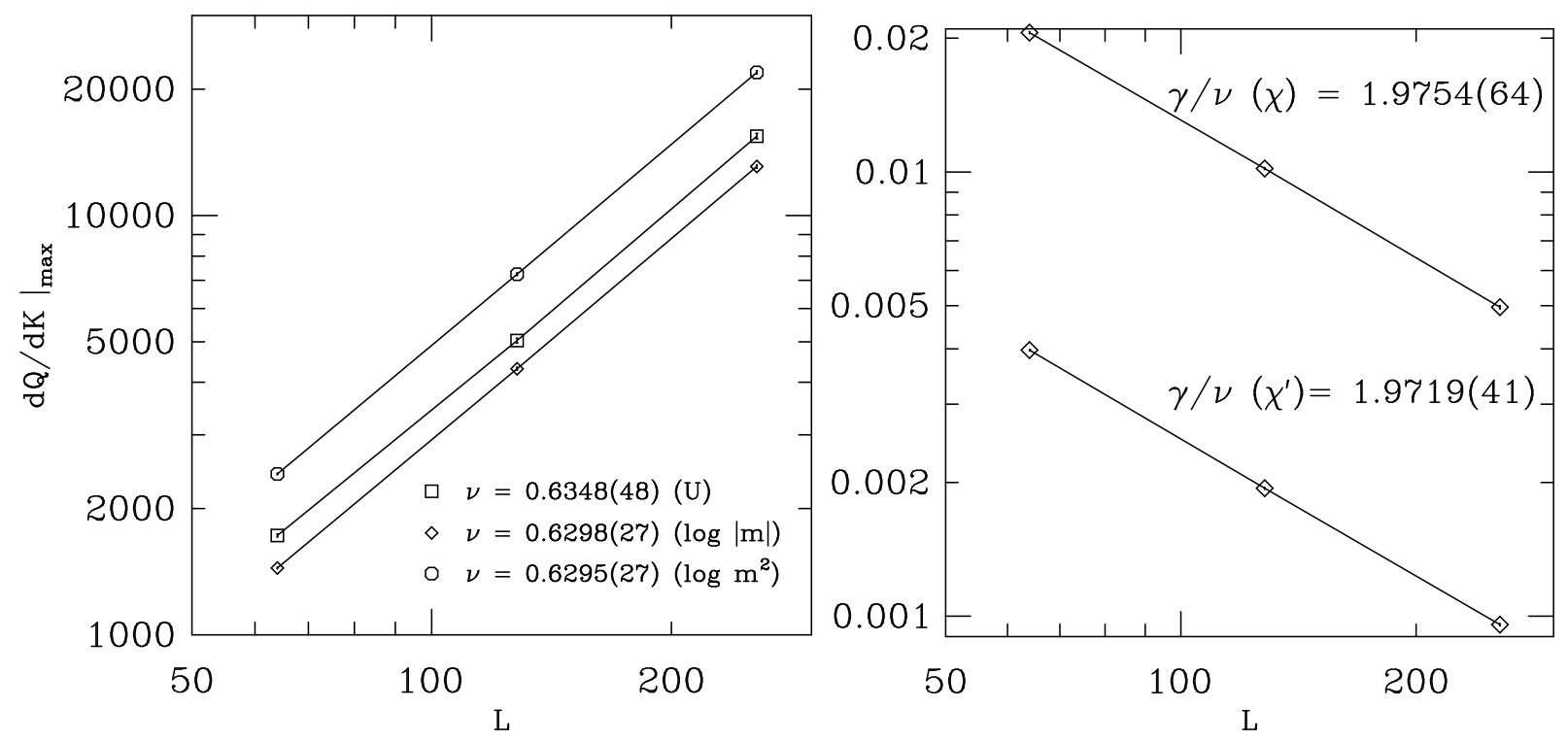

Fig. 3: (A) Estimates of $\nu$ from the finite size scaling of the maxima of the derivatives of $U, \log m$ and $\log m^{2}$ with respect to $K$. (B) The slope of $\log \chi$ versus $\log L$ gives the exponent ratio $\gamma / \nu$ assuming the leading order FSS relation $\chi \sim L^{\gamma / \nu}$.

Estimate of $\nu$ : This is obtained from the finite size scaling of the maxima of thermodynamic derivatives of $U, \log |m|$, and $\log m^{2}$ [6]. For example, $d\left\langle\log m^{2}\right\rangle / d K=\left\langle m^{2} E\right\rangle /\left\langle m^{2}\right\rangle-\langle E\rangle$ is calculated as a function of $K$ using the histogram $H(E, m)$ to re-weight the data. FSS analysis to extract $\nu$ is done keeping only the 
leading term in the scaling behavior

$$
\left.\frac{d Q}{d K}\right|_{\max }=a L^{1 / \nu}\left(1+b L^{-\omega}+\ldots\right),
$$

as we cannot reliably include correction terms with data at only 3 values of $L$. Linear fits to the maximum value versus $L^{1 / \nu}$ are shown in Fig. 3. The quality of the fits is exceedingly good, and the final results are

$$
\begin{array}{ll}
\nu=0.6348(48) & U \\
\nu=0.6298(27) & \log |m| \\
\nu=0.6295(27) & \log m^{2} .
\end{array}
$$

The values obtained from the derivative of $\log |m|$ and $\log m^{2}$ agree, while that from $U$ is higher by its $1 \sigma$ error estimate. These estimates are higher than the MCRG value by roughly one combined $\sigma$.

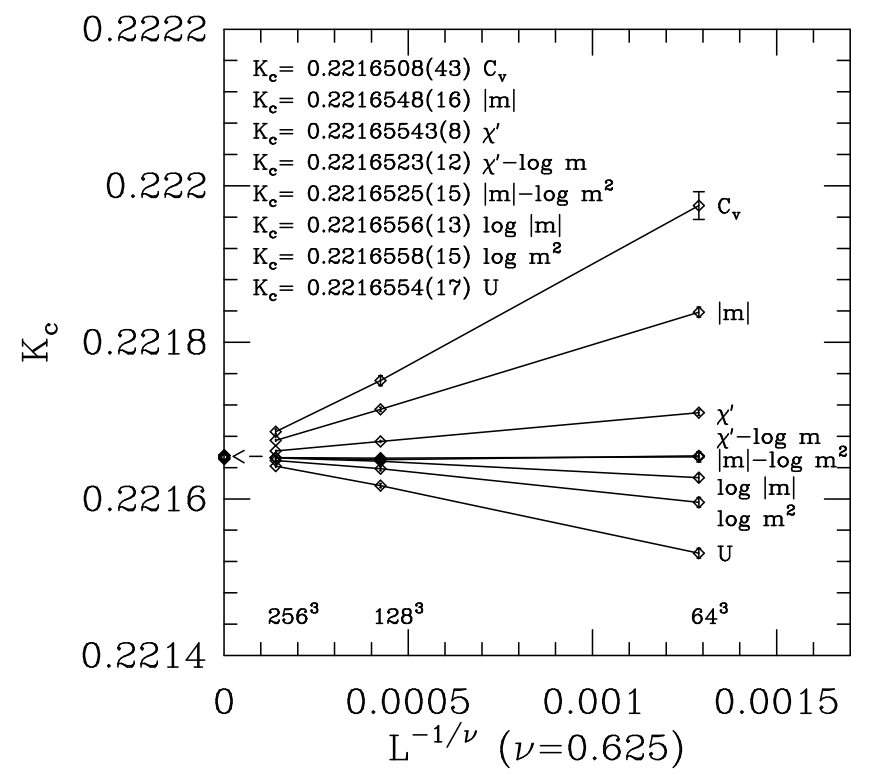

Fig. 4: Estimate of $K_{c}(L)$ obtained from the FSS analysis of different thermodynamic quantities assuming $\nu=0.625$. The data are for $K_{\text {sim }}=0.221655$.

Critical Coupling $K_{c}$ : $K_{c}$ has been calculated in two different ways. One, we considered the location of the maxima of different thermodynamic derivatives as a function of system size. The finite size scaling behavior of these $K_{c}(L)$ is [6],

$$
K_{c}(L)=K_{c}+a^{\prime} L^{-1 / \nu}\left(1+b^{\prime} L^{-\omega}+\ldots\right),
$$

where the values of $a^{\prime}$ and $b^{\prime}$ are different for each thermodynamic quantity. The three data points for each quantity shown in Fig. A have been joined together by straight lines to highlight the deviations from linearity. With just three lattice sizes we cannot include the correction term (i.e. $\omega)$, to take into account the deviations from linearity. In all quantities, the magnitude of these deviations is surprisingly large considering that the data of Ferrenberg and Landau [6] show a good fit to the linear form at smaller $(L=24-96)$ lattice sizes. We do not understand this discrepancy at present other than mention the possibility that in our $64^{3}$ data $\Delta K$ may be too large for the re-weighting to be reliable. Since $\chi^{\prime}$ and $m$ converge from above while $\log |m|$ and $\log m^{2}$ approach it from below, we have constructed linear combinations, $\chi^{\prime}-\log |m|$ and 
$|m|-\log m^{2}$, that show much smaller corrections. As pointed out in [6], the quantity farthest away from $K_{c}$ is $C_{v}$ and it gives $K_{c}$ with the largest errors.

Fig. 国 was generated using $\nu=0.625$. To determine $K_{c}$ we show, in Table 4 , the variation with $\nu$ of $K_{c}$ obtained using the data from $128^{3}$ and $256^{3}$ lattices to extrapolate to $L=\infty$. We find that there is no significant variation with $\nu$ in the canonical range of values $0.62-0.635$. Different observables give $K_{c}$ in the range $0.221653-0.221656$ with a typical statistical error of 0.000002 . For our best estimates we take the mean of the various estimates (excluding that from $C_{v}$ ) with $\nu=0.625$. The result is

$$
K_{c}=0.2216544(20)(15)
$$

where the first error is statistical and the second is an estimate of the systematic error based on the variation with observable type. This value is lower than that obtained by Ferrenberg and Landau [6], but consistent with our MCRG result.

\begin{tabular}{|c|c|c|c|c|}
\hline Observable & $\nu=0.62$ & $\nu=0.625$ & $\nu=0.63$ & $\nu=0.635$ \\
\hline$|m|$ & $0.2216559(22)$ & $0.2216553(22)$ & $0.2216551(22)$ & $0.2216548(23)$ \\
$U$ & $0.2216538(24)$ & $0.2216539(24)$ & $0.2216541(24)$ & $0.2216543(24)$ \\
$\log |m|$ & $0.2216547(17)$ & $0.2216547(18)$ & $0.2216548(18)$ & $0.2216548(18)$ \\
$\log m^{2}$ & $0.2216545(21)$ & $0.2216545(21)$ & $0.2216546(21)$ & $0.2216547(21)$ \\
$C_{v}$ & $0.2216544(58)$ & $0.2216540(58)$ & $0.2216536(58)$ & $0.2216531(59)$ \\
$\chi^{\prime}$ & $0.2216555(10)$ & $0.2216554(11)$ & $0.2216553(11)$ & $0.2216553(11)$ \\
$\chi^{\prime}-\log |m|$ & $0.2216540(17)$ & $0.2216540(17)$ & $0.2216540(18)$ & $0.2216540(18)$ \\
$|m|-\log m^{2}$ & $0.2216529(21)$ & $0.2216529(21)$ & $0.2216529(21)$ & $0.2216529(21)$ \\
\hline
\end{tabular}

Table 4. FSS estimates of $K_{n n}^{c}$ from different observables as a function of $\nu$.

A second estimate of $K_{c}$ is given by the point of crossing of $U(L)$ and $g_{R}(L)$ calculated on two lattices of different sizes. Again, the use of re-weighting trick to extend the data to temperatures in the vicinity of $K_{\text {sim }}$ is essential. Our results are shown in Fig. 5. The final values, taken from the crossing point of $L=128$ and $L=256$ lattices data, are

$$
\begin{aligned}
& K_{c}^{U}=0.2216560 \quad U\left(K_{c}\right)=1.409(9), \\
& K_{c}^{g_{R}}=0.2216551 \quad g_{R}\left(K_{c}\right)=5.23(10) .
\end{aligned}
$$

Note that the error estimates do not take into account the correlations in the data generated by the reweighting. It is interesting to note that the estimate of crossing point obtained from $L=64$ and $L=128$ lattices is $\sim 0.221652$, indicating a convergence from below. Also, the estimates of $K_{c}$ from the two pairs of lattice sizes are in very good agreement with the corresponding results obtained from MCRG analysis.

Critical Exponent $\gamma$ : We estimate $\gamma$ from the finite size scaling of the susceptibility. A linear fit to $\log \chi$ versus $\log L$ is shown in Fig. 3 for data at $K=0.221655$ and for $\mathrm{L}=64^{3}, 128^{3}$ and $256^{3}$ lattices. From the slope we obtain

$$
\gamma / \nu=1.9754(64) \text {. }
$$

(To allow detailed comparison with the results in [6] we also give the value $\gamma / \nu=1.9719(41)$ obtained using $\chi^{\prime}$.) Using the hyperscaling relation $\eta=2-\gamma / \nu$ we now get $\eta=0.0246(64)$. This estimate is consistent with the MCRG result obtained assuming the validity of the linear approximation. We thus quote $\eta=0.025(6)$ as our best value since it covers the various estimates.

\section{Renormalized coupling $g_{R}$ and hyperscaling}

It has been pointed out by Baker and Kawashima [10] that the two limits $L \rightarrow \infty$ and $K \rightarrow K_{c}$ do not commute and that there is a discontinuity in the value of $g_{R}$ when calculated in the following two 

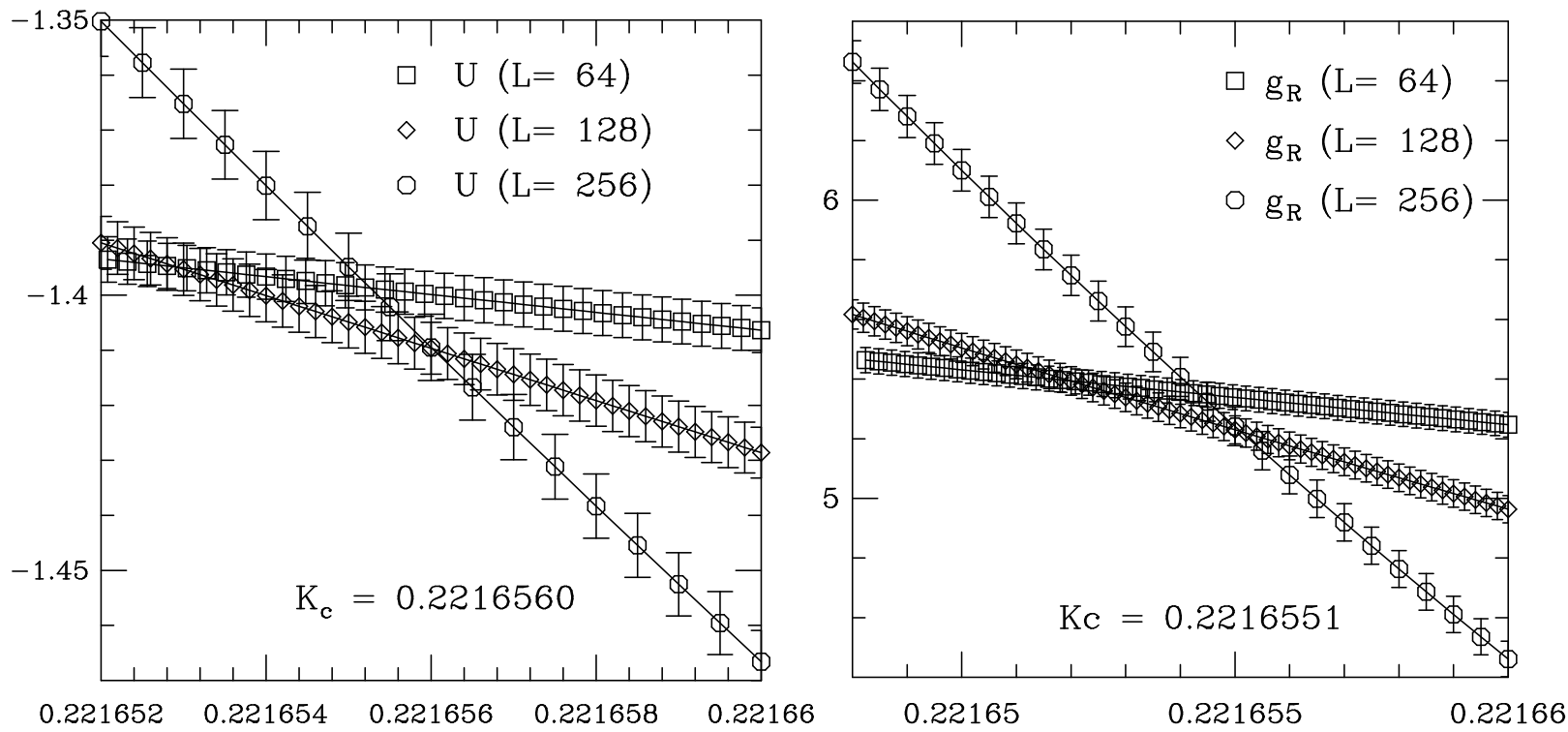

Fig. 5: Plot of $U$ and $g_{R}$ as a function of $K$ obtained from the histogram re-weighting method. The $K_{c}(L)$ estimated from the crossing point of 128 and 256 lattices data is indicated. Note that the values and error estimates are highly correlated.

ways. Our approach, which is to fix $K=K^{c}(\infty)$ and then slowly take the thermodynamic limit versus the "right" way which is to always keep $L / \xi$ large and fixed (the precise value depends on the model and should be representative of the thermodynamic limit) while taking $K \rightarrow K^{c}$. Note that the field-theoretic value $g_{R}=23.73(2)[11$ is an estimate representative of the "right" order of limits. Nevertheless, one can establish hyperscaling by our non-perturbative approach if we can show that $g_{R}$ converges to a non-zero lower bound as $L \rightarrow \infty$.

The data for $g_{R}$ in the vicinity of $K^{c}$ is shown in Fig. 5. One expects an increase in the slope with lattice size as the discontinuity due to the interchange of limits becomes sharper with lattice size. This is borne out by the data. On the other hand the crossing value shows a small decrease between the $128^{3} / 64^{3}$ and $256^{3} / 128^{3}$ lattices, i.e. the data does not converge from below. Based on our data ( 2 crossing points) we guess that $g_{R}(\infty) \approx 5$. We regard this non-zero result as a weaker verification of hyperscaling than one would have liked.

Another necessary condition to test whether hyperscaling holds, on basis of the data for $g_{R}$ in Fig. 同, is whether it is representative of the fixed point value. To check this we have also calculated $g_{R}$ on the blocked lattices. The data on $256^{3} \rightarrow 128^{3} \rightarrow 64^{3} \rightarrow 32^{3}$ lattices is virtually identical, indicating that our estimate does not change along the flow to the fixed point. On smaller lattices the two methods for calculating $\xi$ give different results and the use of finite lattice versus continuum energy-momentum dispersion relation makes a difference. We therefore consider our data on lattices smaller than $32^{3}$ unreliable for the purposes of this test.

\section{Conclusions}

Our new results $K_{n n}^{c}=0.221655(1), \nu=0.625(1)$, and $\eta=0.025(6)$ are an improvement over the previous MCRG values. These values continue to support the notion that the exponents are rational. We have also reconciled the disagreement between finite size scaling and MCRG results for $K_{n n}^{c}$ and $\eta$ by a comparative study using the same data. The value of the exponent $\nu$ from the two methods, however, shows a significant difference. One possible explanation is the lack of various corrections-to-scaling terms in our FSS analysis.

The big surprise of the current MCRG analysis is the result $\omega \approx 0.7$, which is significantly lower than all previous estimates. This issue clearly needs to be investigated further. 
The convergence of $g_{R}^{*}$, defined to be the crossing point value in the limit $L \rightarrow \infty$, seems to be from above. Thus, our data does not provide the desired lower bound to validate hyperscaling. We estimate $g_{R}^{*}(L=\infty)$ from data at the two crossing points to be $\sim 5$. If this non-zero value withstands further scrutiny, then we will have established that hyperscaling holds for the 3D Ising model.

\section{Acknowledgements}

It is a pleasure to thank David Landau and Masuo Suzuki for organizing a very informative workshop in such idyllic surroundings. We thank George Baker and Robert Swendsen for informative discussions. We

are also grateful to the tremendous support provided by the Advanced Computing Laboratory and Thinking Machines Corporation for this project.

\section{References}

[1] C. Baillie, R. Gupta, K. Hawick, and S. Pawley, Phys. Rev. B45 (1992) 10438

$[2]$ G. S. Pawley, R. H. Swendsen, D. J. Wallace and K. G. Wilson, Phys. Rev. B29 (1984) 4030.

[3] H. W. J. Blöte, A. Compagner, J. H. Croockewit, Y. T. J. C. Fonk, J. R. Heringa, A. Hoogland, T. S. Smit and A. L. van Willigen, Physica A161 (1989) 1.

[4] R. Swendsen and J. S. Wang, Phys. Rev. Lett. 58 (1987) 86.

[5] A. M. Ferrenberg and R. H. Swendsen, Phys. Rev. Lett. 61 (1988) 2635.

[6] A. Ferrenberg, and D. Landau, Phys. Rev. B44 (1991) 5081.

[7] B. Freedman and G. Baker, J. Phys. A: Math. Gen. 15 (1982) L715.

[8] H. Blöte, E. Luijten, and J. Heringa, cond-mat/9509016.

[9] S. Zinn and M. Fisher, Maryland Preprint, Nov 1995.

[10] G. Baker and N. Kawashima, Phys. Rev. Lett. 75 (1995) 994.

[11] G. Baker, Quantitative Theory of Critical Phenomena, Academic Press, 1990. 